\title{
Paroxysmal supraventricular tachycardia: improving diagnosis and management within the accident and emergency department
}

\author{
S F O'Rourke, A Sauvage, P A Evans
}

Emerg Med J 2004;21:495-497. doi: 10.1136/emj.2003.012054

Accident and emergency (A\&E) senior house offices (SHOs) have difficulties in interpreting the electrocardiogram (ECG) in narrow complex tachycardia. Correct ECG interpretation is fundamental to patient management in paroxysmal supraventricular tachycardia. This study aimed to assess the ability of A\&E SHOs to interpret the ECG in this setting. Correct diagnosis in $63 \%$ of cases improved to $86 \%$ with the introduction of a standard protocol sheet. Ensuring that $\mathrm{SHO}$ follow agreed guidelines in rhythm recognition and management of PSVT improves patient care.

$\mathrm{T}$ he management of supraventricular tachycardia has been standardised following the introduction of the peri-arrest guidelines by the Resuscitation Council UK. ${ }^{1}$ Studies in the USA both in the prehospital setting, ${ }^{2}{ }^{3}$ and the accident and emergency (A\&E) department, ${ }^{4}$ have shown the difficulties encountered in distinguishing paroxysmal supraventricular tachycardia (PSVT) from other narrow complex tachyarrhythmias.

The correct recognition of arrhythmias is fundamental to patient management. In PSVT the 12 lead electrocardiogram (ECG) will show a regular tachycardia of 150-250 beats per minute with absent $\mathrm{P}$ waves and a narrow QRS complex $(<0.12 \mathrm{~ms}) .^{5}$ There is no evidence to date of A\&E doctors' efficacy in correctly diagnosing and managing patients with this arrhythmia in the UK.

The primary objective of this study was to assess the ability of junior doctors to correctly diagnose PSVT with the use of a prospective audit. The secondary objective was to establish whether the use of a management protocol, completed in all cases of suspected PSVT at the time of treatment, would improve diagnosis and treatment.

\section{METHOD}

This study was conducted in the A\&E department of the Leicester Royal Infirmary serving a population of over one million and with an attendance of about 90000 adults each year. All senior house officers (SHOs) within the department undergo a two week induction programme, which includes a lecture on rhythm recognition and an abbreviated Advanced Life Support (ALS) course. The opinion of senior medical staff is available within the department at all times. Middle grade staff are all ALS providers or instructors. A prospective audit was performed over a 15 month period of all adults who presented with a presumed diagnosis of PSVT based on a 12 lead ECG that was repeated after treatment. A total of 48 SHOs worked in the department over this time period.

The use of vagal manoeuvres and all drugs were recorded within the audit sheet and the peri-arrest guidelines of the Resuscitation Council UK ${ }^{1}$ were clearly visible within the resuscitation room. To ensure all patients were included a search was performed of the resuscitation room log and the patient administration system.

In the subsequent six months a standard protocol sheet was introduced (available to view on line at http://www. emjonline.com/supplemental) and prospectively audited. No additional training was given during the study period that involved 32 SHOs. The protocol sheet instructed the doctor to carefully inspect the ECG for regularity, rate, absence of $\mathrm{P}$ waves, and width of the QRS complex. If the diagnosis was considered to be PSVT treatment was started and recorded on the protocol sheet. ECGs were assessed by a cardiologist blinded to patient treatment. Ethical approval was not required as this was an audit of current practice. Comparison of categorical data was carried out using the $\chi^{2}$ test with a $\mathrm{p}$ value $<0.05$ considered significant.

\section{RESULTS}

Altogether 105 episodes of suspected PSVT were identified in the first audit. In eight patients no ECG could be found and four had spontaneously reverted before full assessment. Of the remaining 93 episodes the final diagnosis was PSVT in 58 (63\%), atrial fibrillation in $22(24 \%)$, atrial flutter in 7 (7\%), ventricular tachycardia in $2(2 \%)$, and sinus tachycardia in 4 (4\%) (table 1).

After the introduction of the standard protocol sheet, a further 48 episodes of suspected PSVT were treated. Two patients spontaneously converted before treatment and ECGs were not found in a further three patients. Of the remaining 43 cases the final diagnosis was PSVT in 37 (86\%), atrial fibrillation in $3(7 \%)$, atrial flutter in $2(5 \%)$, and ventricular tachycardia in 1 (2\%) (table 1 ). There was therefore a $23 \%$ improvement in arrhythmia diagnosis $(95 \%$ confidence intervals: $9 \%$ to $38 \%$ ). The baseline characteristics of each group of patients were similar in terms of age and sex. Sixty five of the 93 patients in the first audit and 27 of the 43 in the second audit had a past history of palpitations/PSVT $(\mathrm{p}=0.72)$.

In the first arm of the audit loop 84 of the 93 patients received adenosine. In 32 cases (38\%) the diagnosis was incorrect. Of the 43 patients in the second study group 6 of the 37 patients who received adenosine were not in PSVT ( $14 \%)$. When both groups of patients are considered together a total of 95 patients had a final diagnosis of PSVT. Ten patients $(11 \%)$ responded to vagal manoeuvres, $74(78 \%)$ to adenosine and the remaining $11(11 \%)$ to verapamil or DC cardioversion (table 2).

The common side effects of flushing, dyspnoea, and chest pain were reported in $17 \%$ of cases. Adenosine was administered to eight asthmatic patients with no adverse

Abbreviations: SHO, senior house officer; PSVT, paroxysmal supraventricular tachycardia; ECG, electrocardiogram 
Table 1 Comparison of final rhythm diagnosis

\begin{tabular}{lll}
\hline Rhythm & Group 1 & Group 2* \\
\hline PSVT & $58(63)$ & $37(86)$ \\
AF/Aflu & $29(31)$ & $5(12)$ \\
VT & $2(2)$ & $1(2)$ \\
ST & $4(4)$ & 0 \\
Total & 93 & 43
\end{tabular}

*After the introduction of a standard protocol sheet. Percentages shown in parentheses.

effects. No patient given adenosine for atrial fibrillation or atrial flutter experienced proarrhythmic side effects. One patient given adenosine for PSVT developed ventricular standstill for six seconds without complication.

\section{DISCUSSION}

The results of this study illustrate the difficulties encountered in the diagnosis of narrow complex tachyarrhythmias by doctors in the A\&E department. It also shows the value of audit and closing the audit loop. Although a mis-diagnosis rate of $37 \%$ would seem to be high it is consistent with a prospective study by Marco et $a l^{4}$ in the US. Of 79 patients treated with adenosine for presumed PSVT within their A\&E department, 46 (58\%) had this as their final diagnosis. The commonest difficulty was in patients with fast atrial fibrillation (14\%) or atrial flutter (7\%). An in-hospital study of 100 patients found only 33 with a final diagnosis of PSVT and 42 with atrial fibrillation or atrial flutter. ${ }^{6}$

Prehospital based studies demonstrate that paramedics with appropriate training and following set protocols are more successful at making the correct diagnosis using "Lead II" rhythm strips. Brady et al identified 139 patients treated with adenosine by paramedics for presumed PSVT. In 105 episodes $(75.5 \%)$ the diagnosis was correct though 18 (13\%) were in atrial fibrillation. In a study of similar design 385 of 107 patients $(79 \%)$ were correctly diagnosed and $15(14 \%)$ were in atrial fibrillation/flutter. The success of close adherence to the treatment protocol, designed to ensure those with fast atrial fibrillation were excluded, explains the $23 \%$ improvement in diagnosis found in this study.

Adenosine has a comparatively high frequency of side effects. Peripheral vasodilatation results in flushing, ${ }^{7}$ carotid body chemoreceptor activation causes dyspnoea, ${ }^{8}$ and chest pain may result from activation of adenosine pain receptors in the heart. ${ }^{9}$ These unpleasant effects are brief reflecting the drugs short half life as it is deaminated to inosine in the plasma and taken up by red blood cells. ${ }^{10}$ Though rare, there are many case reports of adenosine induced arrhythmias. In atrial fibrillation transient slowing of AV nodal conduction after administration of the drug may be of diagnostic benefit but enhanced conduction with a 1:1 ventricular response may occur. ${ }^{11-13}$ A similar response has been described in atrial flutter. ${ }^{14-16}$ This "rebound" phenomenon may result from an increase in sympathetic nerve activity and plasma catecholamine levels. ${ }^{11}$ Adenosine induced ventricular arrhythmias including ventricular tachycardia, ${ }^{17}$ polymorphic ventricular tachycardia, ${ }^{18-21}$ and ventricular fibrillation ${ }^{22}$ have all been described in the literature. Improvements in diagnostic accuracy during the study period resulted in a $24 \%$ decline in the administration of adenosine to patients not in PSVT.

The influence of middle grade staff may have introduced bias in the study. The use of the protocol sheet may have lead to the SHO managing the patient to seek more experienced help more often. We believe that this does not invalidate the results as one of the roles of the protocol sheet is to raise awareness of the difficulties sometimes encountered in making the diagnosis of PSVT. It encourages SHOs to ask
Table 2 Treatment of patients with PSVT

\begin{tabular}{lll}
\hline Intervention & Group 1 & Group 2* \\
\hline CSM & $4(7)$ & $1(3)$ \\
Valsalva & $2(3)$ & $3(8)$ \\
Adenosine & $44(76)$ & $30(81)$ \\
Other & $8(14)$ & $3(8)$ \\
Total & 58 & 37 \\
\hline
\end{tabular}

*After the introduction of a standard protocol sheet. CSM, carotid sinus massage. Percentages shown in parentheses.

themselves relevant questions before starting treatment or to seek help if unsure. If improved patient management in part resulted from increased middle grade input into their care then the protocol sheet has served its purpose.

It was not possible to control for the varying experience of the different groups of SHOs who participated in this study. However the process of SHO selection, the number in training or in "stand alone" posts did not change during the study period.

In conclusion, doctors within the A\&E department have difficulty differentiating PSVT from other tachyarrhythmias, especially fast atrial fibrillation. The use of a standard protocol sheet to be completed during the time of treatment improves rhythm recognition and patient care.

\section{ACKNOWLEDGEMENTS}

The authors would like to thank the medical and nursing staff of the Leicester Royal Infirmary.

\section{CONTRIBUTORS}

Sean O'Rourke designed the standard protocol sheet, participated in data collection, analysis, and is the primary author of the paper. Alain Sauvage initiated the study, participated in data collection, analysis, and writing the paper. Adrian Evans had the original idea, coordinated the study, and participated in writing the paper. Sean O'Rourke acts as guarantor of the paper.

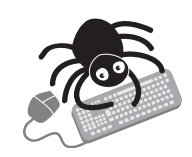

Please visit the journal web site (http://www. emjonline.com/supplemental) to view the protocol sheet.

\section{Authors' affiliations}

S F O'Rourke, Accident and Emergency Department, Tralee General Hospital, Tralee, Co Kerry, Republic of Ireland

A Sauvage, Accident and Emergency Department, West Suffolk Hospital, Bury St Edmonds, Suffolk, UK

P A Evans, Accident and Emergency Department, Morriston Hospital, Swansea, Wales

Funding: none.

Conflicts of interest: none declared.

Correspondence to: Dr S F O'Rourke, Accident and Emergency Department, Tralee General Hospital, Tralee, Co Kerry, Republic of Ireland; orourkes@shb.ie

Accepted for publication 27 October 2003

\section{REFERENCES}

1 Resuscitation Council (UK). Advanced life support manual. 4th ed. London: RCUK, 2000.

2 Brady WJ Jr, DeBehnke DJ, Wickman LL, et al. Treatment of out-of-hospital supraventricular tachycardia: adenosine vs verapamil. Acad Emerg Med 1996;3:574-85.

3 Gausche M, Persse DE, Sugarman T, et al. Adenosine for the prehospital treatment of paroxysmal supraventricular tachycardia. Ann Emerg Med 1994; 24:183-9.

4 Marco CA, Cardinale JF, Adenosine for the treatment of supraventricular tachycardia in the, eds. Am J Emerg Med 1994;12:485-8. 
5 Bar FW, Brugada P, Dassen WRM, et al. Differential diagnosis of tachycardia with narrow QRS complex (shorter than 0.12 seconds). Am J Cardio 1984;54:555-60.

6 Knight BP, Zivin A, Souza J, et al. Use of adenosine in patients hospitalised in a university medical center. Am J Med 1998;105:275-80.

7 Bush A, Busst CM, Clarke B, et al. Effect of infused adenosine on cardiac output and systemic resistance in normal subjects. Br J Pharmacol 1989;27:165-71.

8 Watt AH, Reid PG, Stephens MR, et al. Adenosine-induced respiratory stimulation in man depends on site of infusion. Evidence for an action on the carotid body? Br J Clin Pharmacol 1987;61:779-86.

9 Crea F, Pupita G, Galassi AR, et al. Role of adenosine in pathogenesis of anginal pain. Circulation 1990;81:164-72.

10 Moser GH, Schrader J, Deussen A. Turnover of adenosine in plasma of humans and dogs. Am J Physiol 1989;256:C799-806.

11 Wilbur SL, Marchlinski FE. Adenosine as an antiarrhythmic agent. Am J Cardiol 1997;79:30-7.

12 White RD. Acceleration of the ventricular response in paroxysmal lone atrial fibrillation following the injection of adenosine. Am J Emerg Med 1993; 11:245-6.

13 Hayes BE. Two deaths after prehospital use of adenosine. J Emerg Med 2001;21:151-4.
14 Brodsky MA, Hwang C, Hunter D, et al. Life threatening alterations in heart rate after the use of adenosine in atrial flutter. Am Heart Journal 1995; 130:564-71.

15 Rankin AC, Rae AP, Houston A. Acceleration of ventricular response to atrial flutter after intravenous adenosine. Br Heart J 1993:69:263-5.

16 Slade AKB, Garratt CJ. Proarrhythmic effect of adenosine in a patient with atrial flutter. Br Heart $J$ 1993;70:91-2.

17 Hingorani AD, Foley TH. Non-sustained broad-complex tachycardia following intravenous adenosine. Emerg Med J 1995;12:208-9.

18 Wesley RC, Turnquest $P$. Torsades de pointes after intravenous adenosine in the presence of prolonged QT syndrome. Am Heart J 1992; 123:794-6.

19 Harrington GR, Froelich EG. Adenosine-induced torsades de pointes. Chest 1993;103:1299-301.

20 Smith JR, Goldberger JJ, Kadish AH. Adenosine induced polymorphic ventricular tachycardia in adults without structural heart disease. Pacing Clin Electrophysiol 1997;20:743-5.

21 Kaplan IV, Kaplan AV, Fisher JD. Adenosine induced atrial fibrillation precipitating polymorphic ventricular tachycardia. Pacing Clin Electrophysiol 2000;23:1 40-1.

22 Ben-Sorek ESW, Wiesel J. Ventricular fibrillation following adenosine administration. Arch Intern Med 1993;153:2701-2. 